\title{
FORMACIÓN DE ROBOTS MÓVILES MEDIANTE EL USO DE CONTROLADORES
}

\author{
Adriana Riveros Guevara \\ Ingeniera Mecatrónica \\ Asistente Graduada \\ Universidad Militar Nueva Granada \\ u1801128@unimilitar.edu.co
}

\author{
Leonardo Enrique Solaque Guzmán \\ Docteur de L'INSA Specialite Systemes Automatiques \\ Docente Tiempo Completo \\ Universidad Militar Nueva Granada \\ leonardo.solaque@unimilitar.edu.co
}

(Tipo de Artículo: Reflexión. Recibido el 6/11/2013. Aprobado el 30/12/2013)

\begin{abstract}
RESUMEN
El manejo de sistemas multi-robots, ha adquirido gran atención en las últimas décadas, destacándose en aplicaciones tales como la elaboración de mapas, el transporte de objetos e incluso el rescate de personas. Teniendo en cuenta esta motivación, se realizó la búsqueda de trabajos que abordaron el tema, encontrando diferentes tipos de controladores aplicados a estos sistemas manteniendo la configuración líder-seguidor.
\end{abstract}

Palabras clave

Líder-seguidor, regulador, robot no holonómico, sistemas multi-agente.

\section{MOBILE ROBOT PRODUCTION BY USING CONTROLLERS}

\begin{abstract}
The management of multi-robot systems has gained great importance in recent decades, mainly in applications such as mapping, object transportation and even people rescue. Based on this fact, the search of works on the topic was made, different types of controllers applied to these systems were found and they use the leader-follower configuration
\end{abstract}

\section{Keywords}

Leader-follower, controller, nonholonomic robot, multi-agent systems.

\section{PRODUCTION DE ROBOTS MOBILES EN UTILISANT DES RÉGULATEURS}

\section{RÉSUMÉ}

La gestion de systèmes multi-robots a pris une grande importance dans les dernières décennies, principalement dans applications comme la cartographie, le transport d'objets et même dans le sauvetage de personnes. Par conséquent, en conformité avec ce qu'on a déjà mentionné, on a cherché des travaux au sujet de la production de robots mobiles et on a trouvé des différents types de régulateurs appliqués à ces systèmes qui utilisent la configuration leader-disciple.

\section{Mots-clés}

Leader-disciple, régulateur, robot non-holonome, systèmes multi-agent. 


\section{INTRODUCCIÓN}

El control aplicado a la formación de robots viene siendo estudiado y aplicado por varios investigadores, quienes han visto notables ventajas en implementar un grupo de robots y no un solo robot. Entre las argumentaciones del uso de estos sistemas, se encuentran la reducción de costo a la hora de realizar una tarea o misión, la robustez, la eficiencia energética y el mejoramiento en desempeño [1], entre otros. El movimiento de un grupo de robots, puede ser útil en tareas tales como: manipulación y transporte de objetos largos y pesados, exploración, construcción de mapas en territorios desconocidos, vigilancia y operaciones de seguridad, búsqueda y misiones de rescate, e incluso es ventajoso para ayudar a las personas en estudios de comportamiento social y biológico [2]. Existen diversas formas de manejar las agrupaciones de robots, entre estas, se pueden nombrar: 1) estructura virtual, 2) comportamiento grupal y 3) configuración líder-seguidor, una de las más usadas. Esta última ha sido empleada para realizar estrategias de control como: Backstepping, control por modos deslizantes de primer orden y control por retroalimentación [3]. A continuación se realiza una descripción de los trabajos realizados que involucran técnicas de control usadas para la formación de robots móviles con ruedas, en especial con configuraciones diferencial y omnidireccional.

\section{ESTADO DEL ARTE DE LA FORMACIÓN DE ROBOTS}

El uso de sistemas multi-agente ha crecido notablemente en los últimos años ya que facilita tareas como el hallazgo de personas en apuros, la realización de mapas, el transporte de materiales peligrosos para el ser humano, entre otros.

Para llevar a cabo la formación de robots múltiples se han propuesto principalmente las siguientes técnicas:

- Estructura virtual: trata toda la formación como una única estructura rígida virtual.

- Comportamiento grupal: varios comportamientos deseados son asignados para cada robot, y la acción final de cada robot es derivada mediante la ponderación de la importancia relativa de cada comportamiento [4].

- Líder-seguidor: uno de los robots es designado como el líder, el resto como seguidores. Los robots seguidores tienen que posicionarse con relación al líder manteniendo una posición relativa deseada [5].

La mayoría de aplicaciones desarrolladas, presentan una configuración líder-seguidor para el grupo de robots terrestres, sin embargo hay diversas técnicas para su control tales como $\mathrm{H} \infty$, Modos deslizantes, Backstepping, controles adaptivos, predictivos, etc.
Dichas técnicas han sido desarrolladas mayoritariamente en el exterior y probadas principalmente en entornos de simulación.

Entre las técnicas de control adaptivo, podemos mencionar las encontradas en [6] y [7]. En [6] se realiza la simulación del comportamiento de un sistema con múltiples robots de ruedas con locomoción diferencial, tomando como base el modelo cinemático y usando un control de tipo ADSC (Adaptive Dynamic Surface Control) que permita el manejo efectivo del robot pese a la pérdida de algunos actuadores teniendo en cuenta la efectividad del sensor. Adicionalmente, se hace uso de la teoría de estabilidad de Lyapunov para verificar el comportamiento del sistema en lazo cerrado. En [7] se ejecuta una aplicación en hardware con doce robots móviles, los cuales manejan una comunicación mediante LEDs y fotodetectores. Para realizar el control de este tipo de sistemas, que tienen como principales objetivos el seguimiento de una formación, además de la evasión de obstáculos, se ejecuta un algoritmo de gradiente de fase basado en la naturaleza, especialmente en microorganismos como la ameba, que pueden cumplir sus funciones a pesar de perder alguno de sus miembros. Para llevar a cabo el algoritmo se hace uso de osciladores, los cuales van a generar una transmisión desde altas hasta bajas frecuencias y el cual va a originar el direccionamiento de los robots.

El segundo tipo de control, se puede ver claramente en [5], en donde se realiza un control de tipo integrador Backstepping para un conjunto de robots móviles con locomoción de tipo triciclo. Para la ejecución de este control, se lleva a cabo el modelado teniendo en cuenta coordenadas cartesianas que según los autores, llevan a una estabilidad global del sistema a diferencia de las comúnmente usadas coordenadas polares. Para la evaluación de dicha estabilidad, se hace uso de los teoremas de Lyapunov.

Otro de los controles destacados, es el predictivo, muestras de su utilización se puede ver en [8], en donde se implementa un control UMPC (Unscented Model Predictive Control) en un grupo de robots no holonómicos. EI UMPC es un control basado en el MPC (Model Predictive Control), pero a diferencia de este, tiene en cuenta las incertidumbres que se pueden generar al tratar sistemas de robots, tales como el ruido de los sensores y la presencia de obstáculos que no están determinados, y que en este caso se pueden considerar como restricciones probabilísticas que muestran una distribución Gaussiana.

Dentro de las aplicaciones de $\mathrm{H}^{\infty}$ se encuentra lo realizado en [9], en donde se realiza la simulación del seguimiento de las trayectorias por parte de un conjunto de robots con locomoción por ruedas, en el cual se tiene en cuenta en primer lugar, su comportamiento cinemático y dinámico para el hallazgo de las restricciones del movimiento. Para realizar el seguimiento de las trayectorias por parte del conjunto de robots, se hace uso de controladores $\mathrm{H}_{\infty}$ basados 
en quasi-LPV (quasi-Linear Parameter Varying), método que mitiga los efectos del ruido y de parámetros desconocidos del sistema.

El control por modos deslizantes tiene aplicación en las flotillas de robots móviles, uno de los ejemplos de utilización, se encuentra en [3], en donde se implementan modos deslizantes de segundo orden sobre los robots MIABOT (Fig. 1) tomando la configuración líder-seguidor. Entre las ventajas de la técnica se encuentran que elimina la necesidad de una medición o estimación de la velocidad absoluta del líder y permite el control de la formación utilizando sistemas de visión implementados sobre los seguidores.

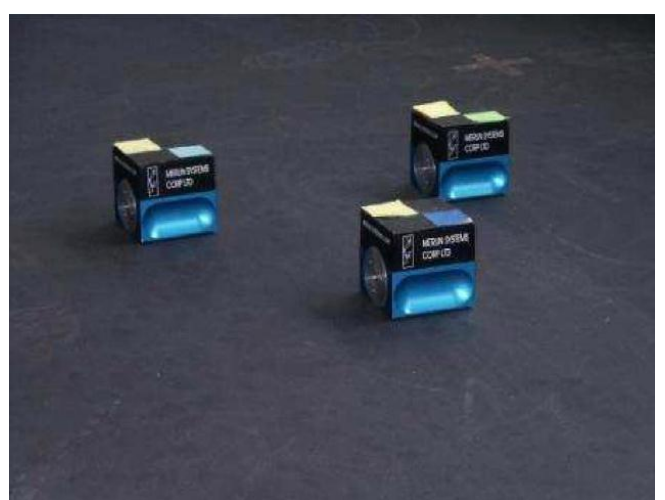

Fig. 1. MIABOT robots [3]

En [2] se realiza un control switcheado y se lleva a simulación tomando tres robots que siguen la configuración líder-seguidor. En el trabajo que se desarrolló, se establece que cualquiera de los robots puede tomar la posición de líder, sin embargo hay que tener en cuenta que no se puede cambiar de rol durante el movimiento a lo largo de la trayectoria. En [10] se maneja el mismo tipo de control, sin embargo esto es llevado a una aplicación real con los prototipos de la Fig. 2. los cuales están instrumentados con láser, cámaras PTZ (Pan,Tilt, Zoom), compás y sónar.

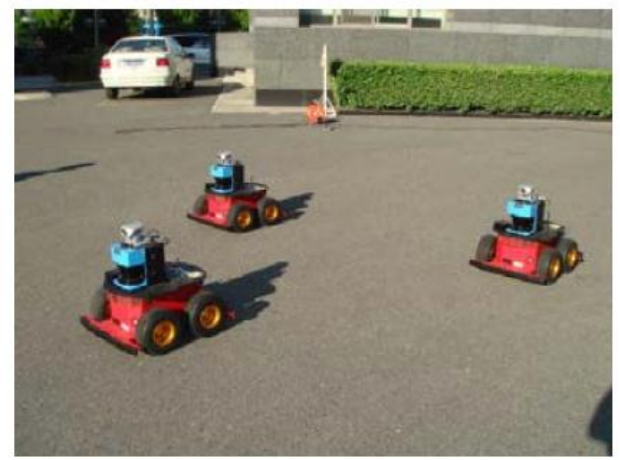

Fig. 2. Robots de prueba para Control Switcheado [10]

Otra de las aplicaciones se puede ver en [1], en donde se realiza la implementación de un control de formación jerárquica basado en un campo vectorial. La formación jerárquica, se muestra como una resolución al problema de rigidez de un sistema de robots que puede generar posibles colisiones tras cambios en las posiciones relativas entre estos debido a la toma de una curva o a un cambio de sección, por lo que se establece un nuevo esquema en el cual hay subgrupos de robots que forman líneas y columnas (Fig. 3. ) y que a su vez minimizan el gasto de recurso en la comunicación con otros robots, ya que solo se comunican los subgrupos y no cada individuo como sería en el caso de una estructura rígida. El campo vectorial sobre el que se basa el algoritmo refleja la ruta a ser seguida por la flotilla de robots a manejar.

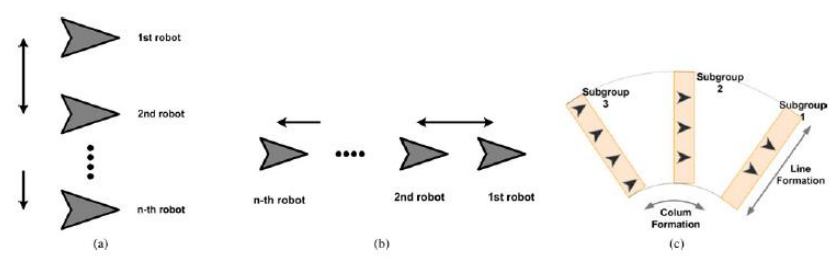

Fig. 3. Estructura jerárquica. (a) Formación en línea. (b) Formación en columna. (c) Formación jerárquica [1]

Otras aplicaciones menos populares pero no menos importantes, se encuentran en [10] y [11]. En [10] se realiza el control de dos robots no holonómicos mediante la configuración líder-seguidor, teniendo en cuenta el error que hay en el seguimiento de la trayectoria deseada, así como los retardos generados especialmente en el seguidor, es por esto que se hace uso del observador de Luenberger, el cual estima el error que será eliminado posteriormente por un controlador de tipo PID o modos deslizantes. En [11] se implementa una configuración triangular con tres robots que presentan locomoción diferencial. La forma de triángulo se establece con el fin de llevar un objeto esférico, para tal objetivo se establece un modo líderseguidor, en el cual el líder es quien esté más cerca a la meta a la cual se quiere llevar el objeto y los demás son seguidores. Para llegar a una conclusión del objetivo se hizo uso de algoritmos PSO (Particle Swarm Optimization). En la Fig. se ve la configuración a la que se llegó mediante simulación.
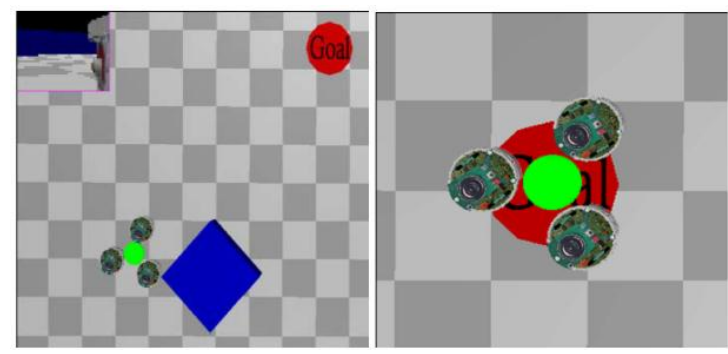

Fig. 4. Simulación de PSO [11]

\section{CONCLUSIONES}

Para la generación y el seguimiento de trayectorias por parte de un sistema de múltiples robots, se han utilizado diversas técnicas, empleando principalmente la configuración líder seguidor, entre los algoritmos más utilizados podemos encontrar Backstepping, modos deslizantes de primer orden y retroalimentación de estados, técnicas que no son tan efectivas tras la pérdida de información de sensores o incluso una aparición repentina de obstáculos. 
Los desarrollos tecnológicos, se han posicionado hoy en día como los principales ayudantes del ser humano en la realización de diferentes labores, un ejemplo de esto, se puede ver tras el uso de agrupaciones de robots móviles con ruedas en labores como: manipulación, transporte de objetos, exploración, construcción de mapas, vigilancia, búsqueda y misiones de rescate. Realizar este tipo de aplicaciones, permite dirigir el enfoque de estos proyectos a un nivel local, ahondando en las necesidades del país y el fortalecimiento de la comunidad científica nacional.

Para que un autómata realice una misión específica, el controlador que lo maneja debe presentar el mejor comportamiento posible, una evaluación de desempeño del regulador, puede significar la reducción de costos tras una disminución en el gasto de energía o incluso una mayor precisión en el procedimiento a ejecutar. De esta manera, la integración entre un controlador de buen desempeño y un grupo de robots terrestres, puede convertirse en una aplicación de interés social, como vigilancia o rescate que despierte el interés de la humanidad en general.

\section{REFERENCIAS}

[1] X. Li, J. Xiao and Z. Cai, "Backstepping Based Multiple Mobile Robots Formation Control" p. 6, 2005.

[2] Y-H. Chang, W-S. Chan, C-Y. Yang, C-W. Tao and S-F Su, "Adaptive Dynamic Surface Control for Fault-Tolerant Multi-Robot Systems". IEEE International Conference on System Science and Engineering, p. 6, 2013.

[3] D. Kurabayashi, T. Choh, J. Cheng and T. Funato, "Adaptive Formation Transition among a Mobile Robot Group based on Phase Gradient" International Conference on Robotics and Biomimetics, p. 6, 2008.

[4] M. Farrokhsiar y H. Najjaran, "An Unscented Model Predictive Control Approach to the Formation Control of Nonholonomic Mobile Robots" 2012 IEEE International Conference on Robotics and Automation, p. 7, 2012.

[5] F. T.B.R, M.H Terra and A.A.G. Siqueira, "Output Feedback Nonlinear $\mathrm{H} \infty$ Control of Wheeled Mobile Robots Formation", 16th Mediterranean Conference on Control and Automation, p. 6, 2008.

[6] M. Defoort, et al., "Sliding-Mode Formation Control for Cooperative Autonomous Mobile Robots", IEEE TRANSACTIONS ON INDUSTRIAL ELECTRONICS, vol. 55, nำ 11, pp. 3944-3954, 2008.

[7] F. Bravo, D. Patiño, K. Melo and C. Parra, "Switching Control and Modeling of Mobile Robots Formation" Robotics Symposium, 2011 IEEE IX
Latin American and IEEE Colombian Conference on Automatic Control and Industry Applications (LARC), p. 6, 2011.

[8] J. Wu and Z. Jiang, "On the Switching Control of Multiple Mobile Robots Formation”, 2009 IEEE International Conference on Robotics and Automation, p. 6, 2009.

[9] J.-W. Kwon and D. Chwa, "Hierarchical Formation Control Based on a Vector Field Method for Wheeled Mobile Robots", IEEE TRANSACTIONS ON ROBOTICS, vol. 28, n 6, p. 11, 2012.

[10]H. Sira Ramirez and R. Castro Linares, "Trajectory tracking for non-holonomic cars: A linear approach to controlled leader-follower formation" 49th IEEE Conference on Decision and Control, p. 6, 2010.

[11]A. Ghaffari and M.R. Esfahanian, "Using Swarm Robots Based on Leader-Followers Method for Spherical Object Manipulation" RSI/ISM International Conference on Robotics and Mechatronics, p. 6, 2013. 